\title{
Designing a Smart Safety Helmet
}

\author{
Rui Wang, Guijun Xiang, Yanping Li, and Samuel Gomes
}

\begin{abstract}
This paper mainly discusses a smart safety helmet project according to certain designing and making methodology to get an entire product. The whole process includes project management, mechanical design, 3D modeling, Arduino software programing etc. And the final product shows that this kind of methodology can achieve the goal of less number of product design modification, the short product development cycle, the low product cost and the high product quality.
\end{abstract}

Index Terms-Arduino program, manufacturing assembly, productive design, project development.

\section{INTRODUCTION}

Any products are becoming more complex to be developed and most of the time require collaborations between various expertise from various enterprises. The interaction and communication between these different extended enterprises are needed to be managed properly so that the appropriate information is given to the appropriate person at the appropriate real time or near real-time.

What is design? Design can be defined as "The process of establishing requirements based on needs, transforming them into performance specification and functions, which are then mapped and converted (subject to constraints) into design solutions (using creativity, scientific principles and technical knowledge) that can be economically manufactured and produced". In traditional craft-based societies, designing or making are not well separated. But in industrial societies, the activity of making (or manufacturing) a product cannot begin as the designing activity that was not completed. Then, the goal of the design process is to describe the product that is to be made, So the end of the design process is to transfer a designed artefact that is ready to be manufactured [1].

We cite a methodology to structure design activities in order to reduce time devoted to routine design process and guide designers to define assembly sequences consistent with the modular products. This paper will focus on the designing and making of product-process information during each phase of product life cycle. We firstly introduce the paper with an art regarding the design and main purpose in this research work. A second part presents the recommended methodology and several software or platform which are required in the

Manuscript received April 20, 2018; revised July 15, 2018. This work was supported by National Natural Science Foundation of China (NSFC) under Grant No. 61771299, 61301027. The authors would like to express our deepest appreciation to all those who provided us the possibility to complete this report.

Rui Wang, Guijun Xiang, Yanping Li are with Shanghai University, China (e-mail: rwang@shu.edu.cn, xiangguijun1@ 163.com, yanpingli@shu.edu.cn).

S. Gomes is with the School of Mechatronics, Methods, Models and Skills Laboratory (M3M), UTBM, Belfort Cedex, France (Corresponding author; e-mail: samuel.gomes@utbm.fr). methodology. Then the procedure will be described on a concrete project example: Designing a smart safety helmet. After a presentation of our product, the last chapter concludes and presents some perspectives for this research work.

\section{DESCRIPTION OF THE METHODOLOGY}

We focus on methodology of designing modular products. In fact, many enterprises have generated all the variants of complete family of modular products according to certain procedure methodology based on developed scientific research work.

Developing its productions must meet customer or market requirements in the design process while being consistent with the assembly constrains, which will help the enterprises remain competitive and successful in the strong competitive economic context. This methodology is a functional knowledge-based engineering and optimization design approach dedicated to routine design. It aims to reduce routine time and optimize procedure to get more time for creativity or innovation. In our project, the methodology involves in the use of several software or platform which are:

ACSP: A popular collaborative PLM (product lifecycle management) platform that can realize the generation of the requirement specification, the engineering bill of materials and so on. Also allows the project member to organize the file management.

BPMN: A standard Business Process Model and Notation, is a standard for business process modeling that provides a graphical notation for specifying business processes based on a flowcharting technique.

ORASSE: Ordered Activities Sequence, a generic software that allows and helps the assembly planner to create easily the product structure, the contact matrix, and the direct graph in order to generate the assembly sequence of the product.

CATIA: A multi-platform CAD (Computer-aided design), CAM (Computer-aided manufacturing) and CAE (Computer-aided engineering) commercial computer software. It is used to design, simulate, analyze, and manufacture products in a variety of industries.

Arduino: sometimes called "electronic building blocks", is an electronic hardware and software platform for interactive product development widely used in the design of electronic system and development of creative product.

\section{Project Description of A SMart SAFety Helmet}

As we mentioned, the main objective of this project is to design a smart safety helmet. During the whole process, we apply certain methodology for the project development. In this section, some research topics are then considered and discussed: requirement specification, external function 
analysis, designing for assembly and programming, digital modelling by $3 \mathrm{D}$ Model and so on.

\section{A. Project Problematic}

The theme of the project is identity to road safety, which is proposed by Samuel GOMES. Accordingly, especially for bicycle safety, we collected some significant information and drew some conclusion.

Firstly, there has large market size for bicycle in China. According to the related data, about 400 million people have bicycles, that is, one out of every three owns a bike. It is worth to point out that there is an explosion of bike rental such as ofo, mobike, etc. The government also actively advocated and promoted bicycle project and vigorously introduced a bike share plan in 2011 to reduce pollution. Secondly, bicycle accidents frequently occurred in China. It is reported that about 350 cyclist fatalities occurred in Shanghai in one year, which accounted for $23 \%$ among all road fatalities. Particularly, using a helmet can reduce fatalities by $80 \%$.

From the above, there exists a huge market of bicycle in China. At the same time, more concern should be taken about the safety of the cyclists. Base on this thoughtfulness, the project of designing a smart safety helmet was put forward

\section{B. Requirement Specification}

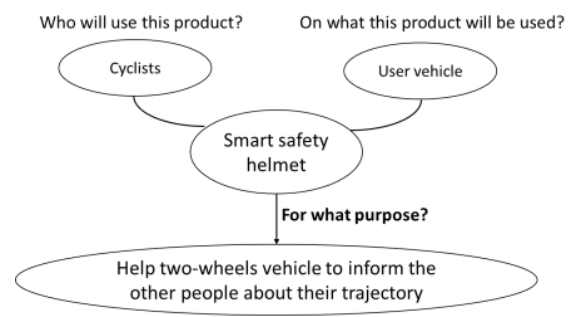

Fig. 1. Main purpose of the project.

To achieve a better match with the needs of the user and reduce the cost, we need to define the purpose or limits of the project and thus establish the framework or ways to check possibility of verification project. Our product is an active safety tool which can provide security and awareness to cyclists on road. The main purpose of this project can be seen in Fig. 1.

\section{Requirement Specification}

Process description plays an important role in achieving project management. The structured analysis and design technique (SADT), a methodology particularly suitable for project-based processed, can help team or managers obtain a better comprehension in what they participate and reach organizational consensus on a management process. The SADT is shown in Fig. 2.

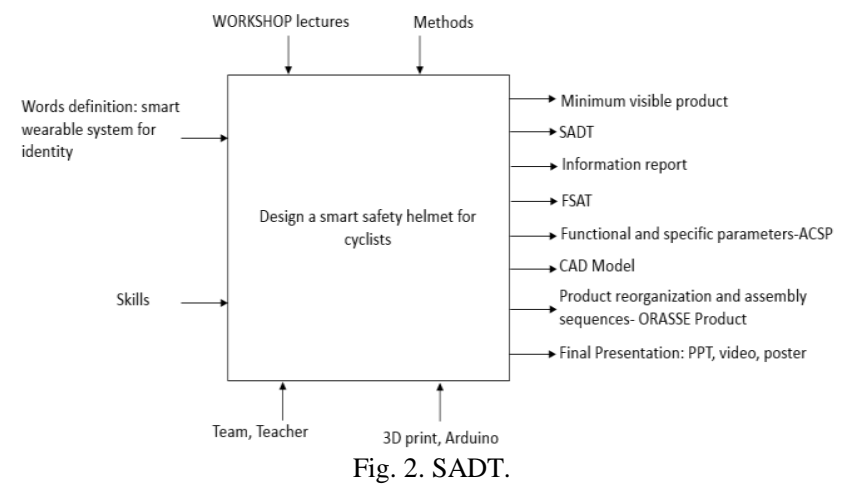

\section{Business Process Model and Notation (BPMN)}

The Business Process Model and Notation can provide us with the capability of understanding our product procedures in a graphical notation, which will facilitate the understanding of the performance collaborations between the operators [2]. Our BPMN shows as follows in Fig. 3.

Fig. 3 shows the project sequence for our product with the target to save time and reduce costs during the design. Then we start by the planning and specification redaction. And we think about how the application could be realized and discuss about something concrete during the meeting. We then do a research about what parameters the application should pilot and so on.

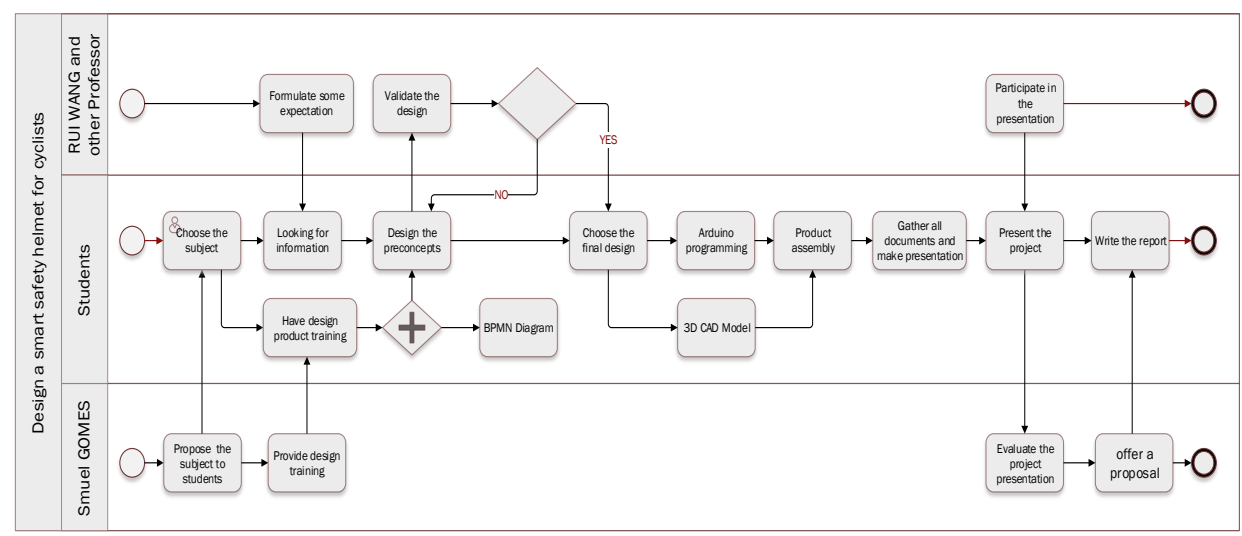

Fig. 3. BPMN

\section{E. External Functional Analysis}

During preliminary design phase, a better attention to products design can impact and bring majors benefits for the manufacturing and assembly phase and then directly keep control of significantly products manufacturing costs. The functional design ensures compliance with the customer requirements for the final product and is able to follow them as fast as possible.

In this section, the project was defined in a technical specification, which can make sure that the project is well managed and converging towards a feasible solution. We studied external function in all different phases, such as usage, design, make and so on. Fig. 4 illustrates one of study phase 
which is the usage phase of the project.

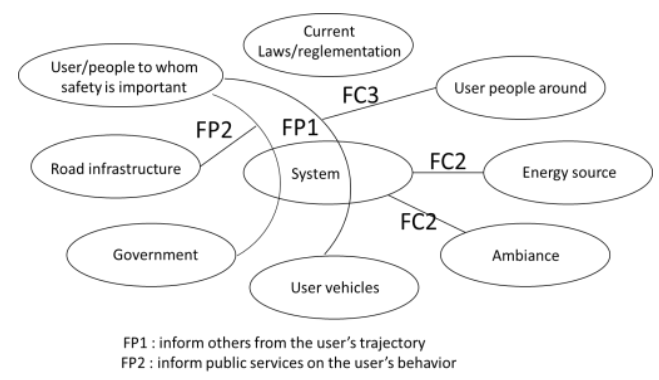

Fig. 4. External functional analysis.

The chart of FU x FU Matrix is shown in Fig. 5. This is an important phase for a product. The functional analysis aims to ensure that the products respect the customer needs and create in a "High Productive" way of new products from a common product basis.

\section{F. FAST Diagram and Engineering Bill of Material}

The Functional Analysis System Technique (FAST) diagram aims to transfer detailed functions to technical functions in order to define some possible solutions principles for a product concept by using a morphological approach. For each phase of the lifecycle, a functional diagram can be created to illustrate interactions between functions of the future product and components of the environment. It composes of the main service function and technical functions of the product as presented in Fig. 5.

\section{TABLE I: FU X FU MATRIX}

\begin{tabular}{lllllll}
\hline \hline & FP1 & FP2 & FC1 & FC2 & FC3 & TOTAL \\
\hline FP1 & & 1 & 1 & 1 & 0 & 3 \\
FP2 & 1 & & 1 & 1 & 1 & 4 \\
FC1 & 1 & 1 & & 0 & 0 & 2 \\
FC2 & 1 & 1 & 0 & & 0 & 2 \\
FC3 & 0 & 1 & 0 & 0 & & 1 \\
\hline \hline
\end{tabular}

After having realized the external functional analysis and also the service and technical function (FAST Diagram) of the product, we need to list all the components that are needed for our product or the engineering bill of materials. All the components and engineering bill of material that we need for the product are listed in the Table II.

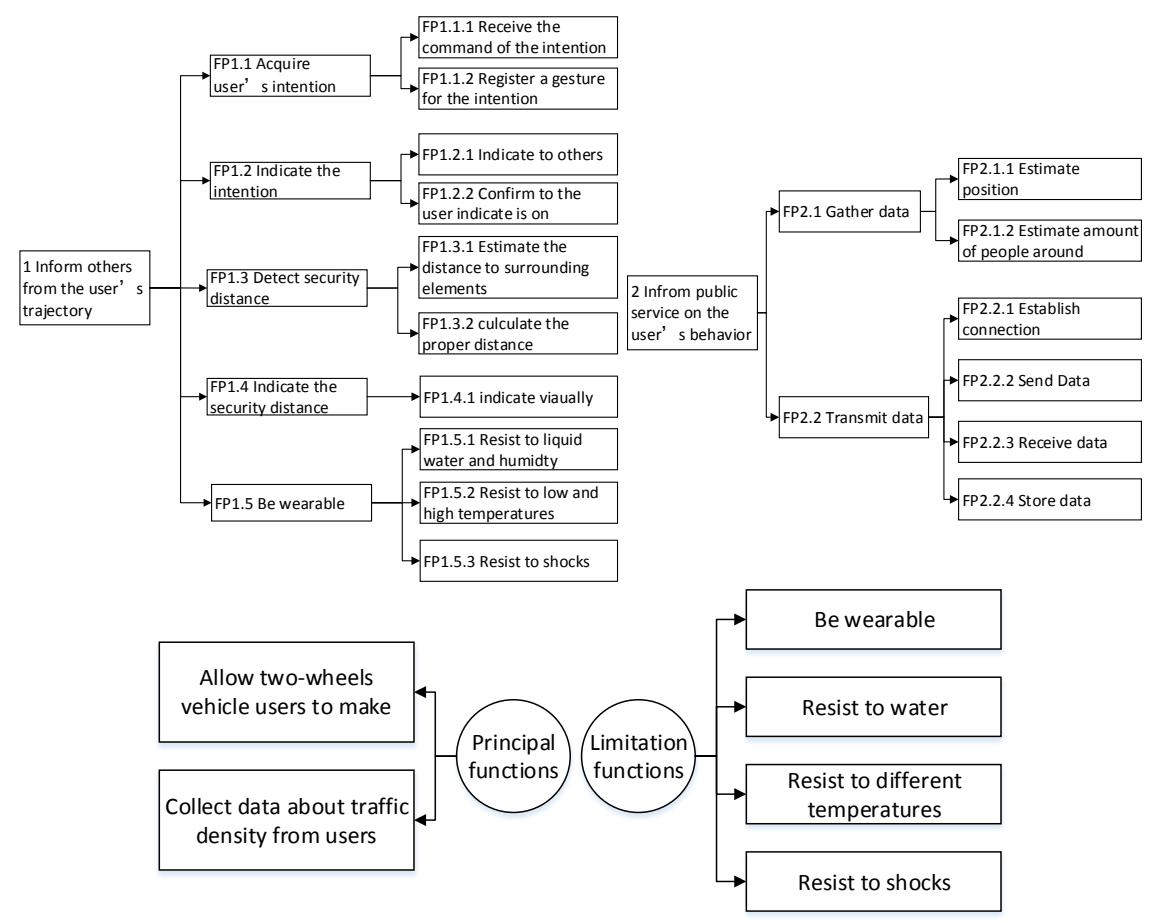

Fig. 5. FAST diagram.

TABLE II: COMPONENTS AND ENGINEERING BILL

\begin{tabular}{llll}
\hline \hline ID & Part Name & QTY & Remark \\
\hline 1 & accelerometer & 1 & 3 degrees of liberty \\
2 & LED band & 3 & Back light for brake, side light for \\
3 & vibrator & 2 & Circular, about $5 \mathrm{~mm}$ radius \\
4 & Distance sensor & 2 & Ultrasound ,40KHz, \\
5 & Arduino & 1 & Controller chip \\
6 & helmet & 1 & supporter \\
7 & battery & 2 & $7.4 \mathrm{~V}-850 \mathrm{mAh}$ \\
8 & relay & 3 & Control the LED band \\
9 & Bluetooth & 1 & Connect the Arduino to smartphone \\
10 & smartphone & 1 & 3 degrees of liberty \\
\hline \hline
\end{tabular}

\section{G. Software and Hardware Design}

This section briefly shows Arduino's hardware and software components. More information about Arduino interactive product design is introduced to readers through the project of a smart safety helmet.

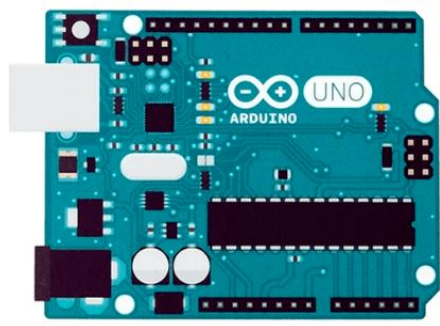

Fig. 6. Uno Arduino board.

The Arduino Uno is a microcontroller board which is based on the ATmega328P and have 14 digital input/output pins, 6 analog inputs, a $16 \mathrm{MHz}$ quartz crystal and so on. It almost 
contains everything needed to support the microcontroller [3].

The development environment to develop the program is the Arduino IDE, which is provided by the Arduino official. It can support Windows, Mac OS and Linux system. When we configure our board, use the Arduino Software (IDE) and start tinker with coding and electronics.

In Arduino programming, solutions can be found on the official website if there have any questions. Our product involves with ultrasonic, LED light, accelerometer, vibrator, relay and so on. Arduino can read the sensor information and automatically control the state of corresponding device to achieve the required functions.

\section{H. 3D CAD Model}

After finished the development of software design, the 3D CAD Model are designed in CATIA V5 environment. CATIA is increasingly chosen as the primary $3 \mathrm{D}$ design system for many companies or developers. CATIA's Design revolutions the way organizations conceive, develop edge through innovative customer experiences [4].

According to the requirements and existing helmet models, we design our helmet 3D model. By using the approach of "Lean Product Development", we are able to pilot certain important geometries by modifying their values to generate a better helmet model. The 3D CAD Model design is displayed in Fig. 7.

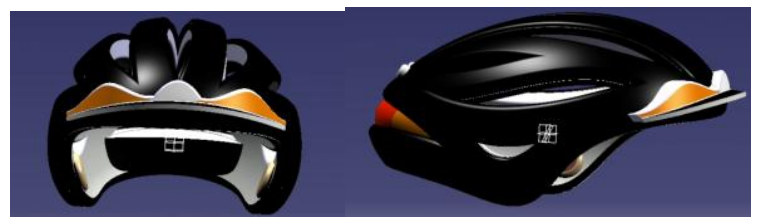

Fig. 7. 3D CAD Model.

\section{Product Architecture and Assembly Sequence}

We should emphasize the fact that product architecture and assembly sequence are both very important in entire process and help increase efficiency and flexibility in the early process. The functional architecture and the generation of the assembly sequence are based on specific algorithms, constituting a reasoning procedure that can be applied in various design domains. Moreover, assembly costs are significant compared to the overall production costs since they can represent more than a half of the direct manufacturing costs. This strengthens the necessity to operate improvements and optimization during this phase of the manufacturing process [5]-[7].

In this section, a generic approach namely "ORASSE" is proposed. It is only focused on this current research work and in accordance with the industrial context of this thesis.

ORASSE Product is linked with ACSP as shown in Fig. 8. It allows the collection of information that has been already in our project in ACSP [8]. We import directly from the ACSP to ORASSE the list of the components of our concept and the functions from the functional analysis. Thanks to ORASSE knowledge we can now obtain easily the contact matrix, the direct graph to generate the best assembly sequence of the product. Taking into account that the functional structure of the product, directed graph, assembly sequence and parameters and rules are relatively simple. Then here is not listed in detail.

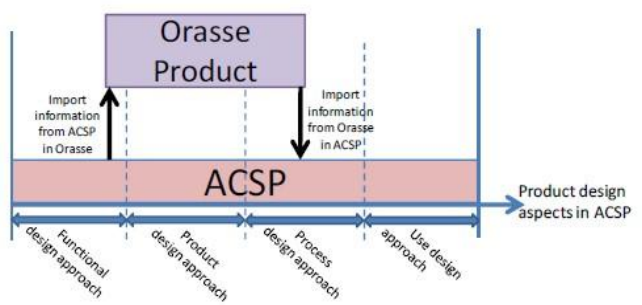

Fig. 8. Interaction between ORASSE and ACSP.

\section{J. Final Product Illustration}

In this chapter, we describe the final result of smart safety helmet and mainly introduce it from two aspects. The final product is given as follows:

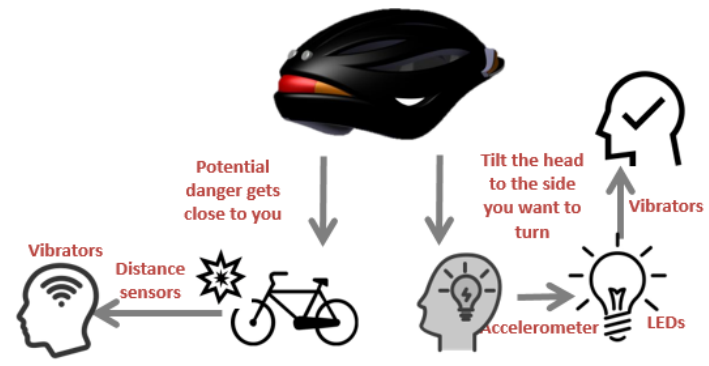

Fig. 9. The system architecture diagram.

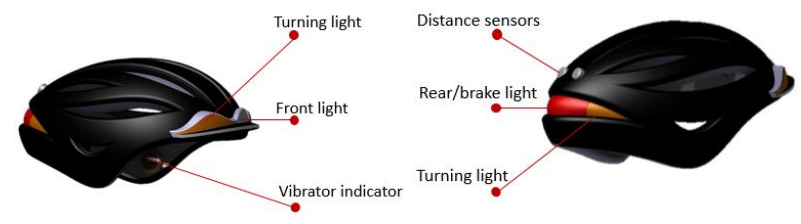

Fig. 10. The font/rear view of the helmet.

Fig. 10 shows that the smart safety helmet can warn cyclists with vibration if someone is dangerously close and the helmet indicates cyclist's direction with LED signalization via a head movement. If we install Bluetooth sensor in the helmet and connect it to our smartphone, the information can be transmitted between smartphone and helmet and finally can be sent to the cloud storage. In addition, we can use phone to confirm our location and know how many people detected around and estimate the crowed density to choose the appropriate route.

\section{K. Future Prospects}

The smart safety helmet product in this paper is upgraded from ordinary helmet combined with various technologies. In the future, we can add more features to the product, such as GPS positioning, audio and video capture, night viewing, etc. which will make the smart safety helmet not only for cyclists but also firefighting and other emergency rescue industries.

\section{CONCLUSION}

This paper focus on the designing and making (Manufacturing and Assembly) in the development cycle of the product and try to create a proactive design in a high productive way. We take into account all the parameters, all the constraints. For this purpose, the project of designing a 
smart safety helmet was done to illustrate and experiment the proposed methodology. Our research approach is structured as follows:

The research begins with problematic project analysis (customer requirements), which should be respected as one of constrains to generate the final product. To help us take into account a maximum of constrains during the early phase and plan all the steps of the product life cycle, requirement specification, SADT and BPMN are described, including customer needs, the beginning of the definition of the product. Then in the external functional analysis and FAST Diagram, the requirements (function) is expressed in spectacular detail which can help the developed products fit as well as possible to the requirements. Then, with ORASSE knowledge, we can manage related parameters behind the items previously defined, easily obtain the directed graph and optimal assembly sequence generated by the goal. Finally, using software platform and materials, we design the 3D CAD Model, achieve programming and get our final product.

\section{REFERENCES}

[1] C. Congram, "How to describe your service: An invitation to the structured analysis and design technique," International Journal of Service Industry Management, vol. 6, no. 6, pp. 6-23, 2015.

[2] D. Monticolo, V. Hilaire, S. Gomes, and A. Koukam, "A multi-agent system for building project memories to facilitate the design process," Integrated Computer-Aided Engineering, vol. 15, no. 1, pp. 3-20, 2008.

[3] G. S. Li, "The application research based on arduino and Kinect," Applied Mechanics \& Materials, pp.635-637, 1742-1745, 2014.

[4] S. Gomes, J. B. Bluntzer and J. C. Sagot, "Functional design through a PLM system for fastening routine definition of CAD models," PLEDM 2006, Virtual Conception, Playa Del Carmen, Mexico, 2006.
[5] S. Gomes, "Coloured graphs for assembly sequences generation applied to modular product ranges," Journal of Mechanical Design, 2012.

[6] X. T. Yan, L. Rivest and S. Gomes, "An assembly oriented design framework for product structure engineering and assembly sequence planning," Robotics and Computer-Integrated Manufacturing, vol. 27, no. 1 , pp. 33-46, 2011

[7] S. J. Wolff, "Statically stable assembly sequence generation and structure optimization for a large number of identical building blocks," Georgia Institute of Technology, 2006.

[8] M. C. Lin, C. C. Lin, Y. Y. Tai, M. S. Chen and C. C. Tseng, "A modularized contact-rule reasoning approach to the assembly sequence generation for product design," Concurrent Engineering, vol. 20, no. 3, pp. 203-221, 2012.

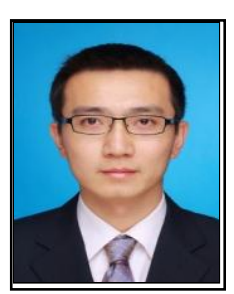

Rui Wang received his B.S. and Ph.D. degree both in electronics and information engineering from Xidian University, Xi'an, Shaanxi, China, in 2004 and in 2009 , respectively. Since then, he has been with the School of Communication and Information Engineering, Shanghai University, where he is currently an associate professor. His research interests include sensor networks, geometric algebra, multimedia signal processing.

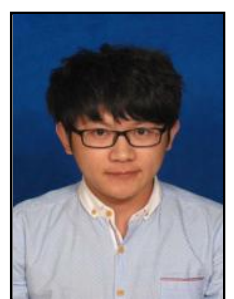

Guijun Xiang received his B.S. degree in communication engineering from Shanghai University, Shanghai, China, in 2016. He is now pursuing the M.S. degree in the School of Communication and Information Engineering, Shanghai University. He is currently committed to electronic and information engineering, internet of things mainly. 